\title{
Dust Masses in Early-Type AGN and Inactive Galaxies
}

\author{
Ramiro D. Simões-Lopes ${ }^{1,2}$, Paul Martini ${ }^{2}$, \\ and Thaisa Storchi-Bergmann ${ }^{1}$ \\ ${ }^{1}$ Instituto de Física, Universidade Federal do Rio Grande do Sul, Av. Bento Gonçalves 9500, \\ Porto Alegre, RS, Brazil \\ Email: ramirosl@if .ufrgs.br \\ ${ }^{2}$ Department of Astronomy and Center for Cosmology and Astroparticle Physics, The Ohio \\ State University, $140 \mathrm{~W}$ 18th Avenue, Columbus, Ohio 43210-1173, USA \\ Email: martini@astronomy.ohio-state.edu
}

Keywords. galaxies: active, galaxies: elliptical, galaxies: ISM, galaxies: photometry, infrared: galaxies, infrared: ISM

It has been known for some time that elliptical galaxies have some form of dust structure at their centers (Kormendy \& Djorgovski 1989; van Dokkum \& Franx 1995). Nevertheless the exact amount and the origin of the dust is still unknown. In a previous paper, we have shown that all early-type galaxies hosting an AGN have dust at their centers as opposed to only a quarter of the inactive galaxies in a pair-matched sample (Simões Lopes et al. 2007). Here we use Spitzer (IRAC and MIPS) observations of the same sample - 32 active and 32 inactive early-type galaxies - and state-of-the-art spectral modelling from Draine \& Li (2007) to estimate the dust mass present in these galaxies. We have also created PAH images by estimating and subtracting the stellar-only contribution to the flux at the $7.9 \mu \mathrm{m}$ image from the $3.6 \mu \mathrm{m}$ image.

We have found that early-type AGN host galaxies typically have $\sim 10^{6} M_{\odot}$ of dust, which is at least 10 times more than that in the matched sample of inactive galaxies with the same luminosity and distance. We have not seen in the Spitzer images any evidence for diffuse dust in galaxies without dust lanes in HST images, meaning that the PAH emission also traces the dust lanes found in the HST data. While the approximately order-of-magnitude difference between the active and inactive galaxies is substantial, the total measured dust masses are sufficiently small that it is consistent with an origin from either mass loss from bulge stars or accretion from small, gas-rich satellites.

\section{References}

Draine, B. T. \& Li, A. 2007, ApJ, 657, 810

Kormendy, J. \& Djorgovski, S. 1989, ARAA, 27, 235

Simões Lopes, R. D., Storchi-Bergmann, T., de Fátima Saraiva, M., \& Martini, P. 2007, ApJ, 655,718

van Dokkum, P. G. \& Franx, M. 1995, AJ, 110, 2027 